\title{
Louis Jouvet. Artisan de la scène, penseur du théâtre, dir. È. MASCARAU et J.-L. BESSON
}

\section{Stefano Genetti}

\section{(2) OpenEdition}

\section{Journals}

\section{Edizione digitale}

URL: https://journals.openedition.org/studifrancesi/32168

DOI: 10.4000/studifrancesi.32168

ISSN: 2421-5856

\section{Editore}

Rosenberg \& Sellier

\section{Edizione cartacea}

Data di pubblicazione: 1 août 2020

Paginazione: 435-436

ISSN: 0039-2944

\section{Notizia bibliografica digitale}

Stefano Genetti, «Louis Jouvet. Artisan de la scène, penseur du théâtre, dir. Ė. mascarau et J.-L. BESSON», Studi Francesi [Online], 191 (LXIV | II) | 2020, online dal 01 septembre 2020, consultato il 16 septembre 2021. URL: http://journals.openedition.org/studifrancesi/32168 ; DOI: https://doi.org/10.4000/ studifrancesi.32168

Questo documento è stato generato automaticamente il 16 septembre 2021.

\section{(c) (i) (9)}

Studi Francesi è distribuita con Licenza Creative Commons Attribuzione - Non commerciale - Non opere derivate 4.0 Internazionale. 


\title{
Louis Jouvet. Artisan de la scène, penseur du théâtre, dir. È. MASCARAU et J.-L. BESSON
}

\author{
Stefano Genetti
}

\section{NOTIZIA}

Louis Jouvet. Artisan de la scène, penseur du théâtre, dir. È. MASCARAU et J.-L. BESSON avec la collaboration de J. HUTHWOHL, K. LE BAIL, J.-L. RIVIÈRE et M. VÉRON, Montpellier, Deuxième époque, 2018, $368 \mathrm{pp}$.

1 Nell'epigrafe, datata 1972, a Spectres, mes compagnons - intensa riflessione sul potere della letteratura in relazione all'esperienza della deportazione consegnata sotto forma di lettera al maestro Louis Jouvet (1887-1951)-, Charlotte Delbo sottolinea l'immateriale eppure inesauribile presenza delle «créatures du poète», spettri più reali delle creature «de chair et de sang»: grazie ad esse, «nous sommes reliés aux autres humains, dans la chaîne des êtres et dans la chaîne de l'histoire» (Paris, Berg International, 1995, p. 7; si veda Charlotte Delbo. Un témoin écrivain et dramaturge, sous la direction de C. Douzou et J.-P. DufIET, Trento, Dipartimento di Lettere e Filosofia, 2016, «Labirinti» 162, 214 pp.). Se agli occhi di Denis Podalydès della Comédie-Française Jouvet rappresenta la figura del «professeur absolu» (p. 353), a riportare quelle righe di Charlotte Delbo (p. 267), mediatrice dell'insegnamento di Jouvet poiché le si deve la redazione delle lezioni parzialmente confluite in Molière et la comédie classique e Tragédie classique et théâtre du XIX ${ }^{\mathrm{e}}$ siècle (È. MASCARAU, Jouvet au Conservatoire. Questionnements et intuitions face à l'écart entre les éditions et les archives, pp. 339-352), è il regista Jacques Lasalle.

Eloquenti in merito all'attualità dell'eredità di Jouvet, sono queste solo due delle numerose testimonianze di professionisti del teatro che si alternano ai saggi di storici e studiosi negli Atti delle tre giornate del convegno tenutosi a Parigi nel marzo 2015: 
insieme, $\mathrm{i}$ trentacinque interventi coltivano un dialogo tra artisti e ricercatori a immagine dell'intrecciarsi di pratica e teoria che ha contraddistinto l'attività di Jouvet (È. MASCARAU, Introduction générale, pp. 7-9). Altrettanto emblematici sono i luoghi che hanno ospitato il colloque: oltre all'Athénée Théâtre, che del suo celebre direttore ha adottato il nome, la Maison de la Radio dove è stata registrata e poi trasmessa da France Culture la Classe Jouvet, esito degli ateliers condotti presso il Conservatoire National d'Art Dramatique a partire dagli scritti sulla preparazione dell'attore, in linea col montaggio Elvire Jouvet-40 realizzato da Brigitte Jaques e basato su sette lezioni riguardanti la scena sesta del quarto atto di Dom Juan (si vedano le pp. 331-333), nonché la Bibliothèque Nationale de France, il cui Département des Arts du Spectacle custodisce il fondo Louis Jouvet esplorato nella maggior parte dei contributi (Mileva STUPAR, Noter la mise en scène, le cas des archives Jouvet, pp. 137-159): copioni annotati, manoscritti, corrispondenze, bozzetti e registri classificati dalla stretta collaboratrice Marthe HERLIN (Naissance au théâtre, pp. 19-27). Di Jouvet, quest'ultima parla come di un difensore e archivista della memoria teatrale, ripercorrendone gli anni di formazione con il Théâtre d'Action d'Art e l'allestimento, nel 1909, dell'École des femmes, precoce indizio di una fedeltà all'autore che sempre rappresenterà per lui il Teatro (sulle ultime messe in scena, si sofferma Jean-Louis BESSON, Jouvet metteur en scène de Molière, "Dom Juan" et "Tartuffe", pp. 219-230, mettendone in luce l'innovatività, fondata su un'accurata documentazione e informata, nel caso di Don Giovanni, da una visione giansenista dell'eroe dinanzi alla morte, scisso tra salvezza e dannazione).

Rubricati in tre sezioni - «Des origines à l'épreuve du plateau», «Louis Jouvet metteur en scène» e «Louis Jouvet acteur et pédagogue»-, i vari contributi ricostruiscono la carriera di Jouvet dall'esperienza giovanile al Vieux-Colombier all'affermazione come attore, regista e professore alla Comédie des Champs-Elysées, all'Athénée e al Conservatoire, dalla fondazione del Cartel in quanto manifestazione di una rinnovata politica culturale (Marc VÉRON, Le Cartel ou le paradoxe protéiforme, pp. 45-57) alla promozione internazionale del teatro francese (mentre nell'Ouverture, Robert ABIRACHED delinea la situazione teatrale nell'immediato secondo dopoguerra, al ritorno di Jouvet dall'esilio sudamericano, un ulteriore intervento di M. VÉRON verte sulle tournées e sull'attenzione riservata al pubblico: Les campagnes de Jouvet et de ses comédiens à travers le monde, pp. 235-248). Da vari punti di vista vengono illuminate le molteplici sfaccettature dell'uomo di teatro: l'ideatore dei dispositivi modulabili atti a ridurre il realismo mimetico delle messe in scena di Copeau (Alice CARRÉ, Construire l'épure: idéaux et paradoxe. La scène architecturée du Vieux-Colombier, pp. 85-100) e il prefatore, nel 1942, dell'edizione francese del trattato di Nicola Sabbattini Pratica di fabricar scene e macchine $n e$ ' teatri (1638), promotore di una visione organicista dello spazio teatrale che, tesa al superamento del conflitto tra possibilità tecniche e necessità drammatiche (Marcel FREYDEFONT, La vérité professionnelle. Louis Jouvet et le sens de la pratique: matérialités de la représentation, une certaine pensée du théâtre, pp. 59-67), investe il processo creativo anche nelle sue implicazioni soggettive ed emotive (Sandrine DUBouilH, Scénographie et «mystère du théâtre», pp. 69-82); il regista pioniere dell'amplificazione della voce, talora preregistrata (lo spettro in Intermezzo di Giraudoux, la parola degli dèi in La machine infernale di Cocteau: Jeanne BOVET, Jouvet et les technologies sonores. Dramaturgies et dispositifs de sonorisation de la voix à la Comédie des Champs-Elysées, pp. 101-114), attento all'orchestrazione della partitura drammatica (Catherine STEINEGGER, Son, musique et mise en scène selon Jouvet, pp. 115-129); l'attore dalla recitazione trattenuta, interiorizzata, 
apprezzata per la minuziosa stilizzazione del personaggio, per l'espressività dei silenzi e dell'immobilità facciale (Marion CHÉNETIER-ALEV, Le jeu de Jouvet à travers la presse, pp. 285-299); il maestro il cui metodo si basa sul respiro della parola, sulla dinamica delle sensazioni e sulle loro ripercussioni fisiche, corporee e gestuali e il teorico del dialogo immaginario Écoute, mon ami e di Le comédien désincarné, vale a dire l'attore che si dissocia da sé ma in parte anche dal ruolo che interpreta affinché incarnazione della parola possa esserci agli occhi del pubblico (Louis DIEUZAYDE, L'acteur, le corps et la langue, pp. 305-316).

4 Accanto ad articoli che rendono conto della collaborazione con Cocteau - parla di regia condivisa Gérard LIEBER in Louis Jouvet et "La machine infernale" de Cocteau (pp. 179-190) e dei sodalizi con Jules Romains - oltre a Knock, la trilogia di commedie incentrate su Le Trouhadec (Olivier RoNY, Jouvet metteur en scène de Jules Romains, pp. 191-204) - e con Giraudoux (Guy TEISSIER, Secrets du théâtre, pp. 207-217), figurano i paralleli con altri praticiens e pensatori del teatro. Si va dalla durevole amicizia con Copeau, non priva di divergenze estetiche dovute, ad esempio, alle concessioni di Jouvet al vedettariat (Marco CoNsolinI, Copeau, Jouvet, qui avait raison?, pp. 29-44) al sostegno assicurato a Mejerchol'd (Béatrice PICON-VALLIN, Louis Jouvet et Vsevolod Meyerhold ou les feuilletons de la création, pp. 161-176), dalle affinità della nozione di sentiment con la memoria affettiva di Stanislavskij (Jean-Loup RIVIÈRE, La leçon de Jouvet, pp. 325-329) alla parentela sotterranea, anche in termini di repertorio, tra Jouvet et Vitez: repères pour une "critique de l'intérieur" (Georges BANU, pp. 257-265). Sui rapporti tra Strehler et le "patron" (pp. 249-255) si concentra Odette ASLAN, evocando le rappresentazioni dell'École des femmes a Roma nel 1948 e di Questa sera si recita a soggetto di Pirandello l'anno seguente a Parigi, le comuni ricerche sull'improvvisazione nella commedia dell'arte e l'entusiasmo di Jouvet e Dullin spettatori dell'Arlecchino servitore di due padroni di Goldoni. Nel 1987, mentre prepara l'allestimento del Don Giovanni di Mozart-sulla copertina del programma della Scala figura Jouvet nei panni di Dom Juan-, Strehler inaugura il Piccolo Teatro Studio con Elvira o della passione teatrale, versione italiana dell'Elvire Jouvet-40 cui si è accennato. Nel preambolo di quello spettacolo, Strehler incarna l'attore che, calato il sipario, solo allo specchio del camerino, ancora truccato e come sospeso «tra il teatro e la vita laica», getta un duplice sguardo su di sé e sul personaggio che interpreta, a sua volta spettatore del proprio destino, condensando così le tensioni tra identificazione e distacco, concreto e ineffabile, effimero e pervasivo che definiscono lo «stato drammatico» secondo Jouvet, suo maestro spirituale. 